\title{
Hipertensão arterial pulmonar: uma proposta multidisciplinar
}

\author{
Pulmonary arterial hypertension: \\ a multidisciplinary proposol
}

\author{
Rogério Rufino* \\ Cláudia Henrique da Costa \\ Bianca Abbud \\ Márcio Macri \\ Esmeralci Ferreira
}

\section{Resumo}

A hipertensão arterial pulmonar (HAP) é uma doença rara caracterizada pelo aumento progressivo na resistência vascular pulmonar, que causa insuficiência cardíaca direita e morte prematura. Durante as últimas décadas, uma melhor compreensão da patogênese da HAP levou à aprovação de várias terapias específicas, que conferem estabilização clínica e recuperação parcial da hemodinâmica, qualidade de vida e sobrevida dos pacientes. As manifestações clínicas da HAP são inespecíficas, mas progressivas. O sintoma de dispneia, o de maior frequência, geralmente tem sido atribuído a outras doenças cardiorrespiratórias mais frequentes, o que leva ao retardo do diagnóstico. Assim, cerca de 75\% dos pacientes com HAP se apresentam na classe funcional III ou IV (escala da New York Heart Association) no momento em que o diagnóstico é estabelecido. O Brasil tem, até o mês de março de 2013, indexados no banco de dados PubMed, 52 artigos sobre este tema desde o início do século XX. Na verdade, a doença foi relevada até o início da década de 1990, quando se verificou que a sua frequência era maior do que se imaginava e que ela poderia ser abordada em conjunto ou isoladamente, nos casos de etiologia idiopática, melhorando a qualidade de vida e aumentando a sobrevida em anos. No Brasil, apesar dos avanços de divulgação terapêutica e diagnóstica, há poucos centros de referência de tratamento e diagnóstico, o que dificulta a capilarização do conhecimento. Esta revisão apresenta o cenário atual do diagnóstico das doenças com HAP e suas amplas perspectivas de tratamento.

Descritores: Hipertensão pulmonar; Terapia ; Revisão.

\begin{abstract}
Pulmonary arterial hypertension (PAH) is a rare disease characterized by a progressive increase in pulmonary vascular resistance, leading to right heart failure and premature death. Over the last decades, a better understanding of the
\end{abstract}


pathogenesis of PAH has led to the approval of several targeted therapies that confer improvements in patients' clinical and haemodynamic status, quality of life and survival. The clinical manifestations are non-specific but progressive, with the majority of patients presenting with worsening dyspnea, the most common symptom, that has usually been attributed to other cardiorespiratory disorders. As a consequence, diagnostic delay is usual, with $75 \%$ of patients reporting New York Heart Association functional class (NYHA-FC) III or IV symptoms by the time the diagnosis is established. Brazil has until March 2013 indexed in the database PUBMED 52 articles on this topic since the early twentieth century. In fact, the disease was forgotten until the early 1990s, when it was found that their frequency was greater than previously thought and that it could be approached together or separately, in cases of idiopathic etiology, improving quality of life and increasing survival in years. In Brazil, despite advances in therapeutic and diagnostic approach, there are few reference centers for diagnosis and treatment, which hinders the capillarization of knowledge. This review presents the current scenario of the diagnosis of $\mathrm{PAH}$ and its broad perspective for treatment.

Keywords: Pulmonary arterial hypertension; Therapy; Review.

\section{Introdução}

A hipertensão arterial pulmonar (HAP) é uma consequência típica de um grupo de doenças com diferenças fisiopatológicas e prognósticas, mas que tem como fator comum a elevação da resistência vascular pulmonar com consequente hipertrofia e falência do ventrículo direito que, se não abordadas de forma específica, evoluem em curto espaço de tempo para óbito. Se após ampla investigação clínica não for encontrada nenhuma causa secundária para a HAP, esta será categorizada como idiopática. ${ }^{1,2}$

Atualmente, a HAP é definida hemodinamicamente e pelo cateterismo cardíaco direito, quando o valor da pressão média da artéria pulmonar for maior ou igual a $25 \mathrm{mmHg}$, com a pressão capilar pulmonar encunhada em ou abaixo de $15 \mathrm{mmHg}$. ${ }^{1,2}$

Das múltiplas causas de HAP, a de etiologia idiopática é a que possui maior dificuldade na abordagem terapêutica e representa o principal desafio clínico.

\section{Epidemiologia}

Estima-se que a HAP idiopática ocorra em torno de 5,9 casos por milhão de habitantes, com incidência de 2,4 novos casos por ano para cada 1 milhão de habitantes. Há um discreto predomínio no sexo feminino (relação de 1,7:1), principalmente jovens na 3 a década. Não há predomínio étnico. Em entre 6 a 10\% dos pacientes com HAP idiopática, a transmissão hereditária tem sido notada (principal mutação relacionada ao gene BMPR-2). Em relação à hipertensão pulmonar associada a outras doenças, a frequência é variável, pois depende da gravidade da condição de base. ${ }^{3}$

\section{Fisiopatologia}

Acredita-se que a HAP idiopática seja o resultado de anormalidades na função endotelial, com redução da síntese de substâncias vasodilatadoras (ex.: prostaciclina e óxido nítrico) e aumento da síntese de substâncias vasoconstritoras (ex.: endotelina). A vasoconstrição prolongada determina o remodelamento da parede vascular, reduzindo fixamente a luz vascular. Existe ainda a possibilidade de alterações no potencial de membrana das células endoteliais, por alteração nos canais de potássio e do músculo liso, o que determinaria um maior tônus vascular. ${ }^{4}$ Entre os 6 a $10 \%$ dos pacientes que possuem predisposição familiar, uma investigação mais apurada de um possível comprometimento genético de transmissão autossômica dominante com penetração incompleta deve ser feita.

\section{Classificação}

A classificação da HAP tem sido sistemati- 
camente atualizada. A última ocorreu em 2008, na IV Conferência Mundial em Hipertensão Pulmonar realizada em Dana Point, EUA (quadro 1$).^{1,2}$

\section{Diagnóstico}

O diagnóstico da HAP é baseado na anamnese, exame físico e exames complementares (invasivos e não invasivos).

\section{Apresentação clínica}

Os sintomas dos pacientes com HAP são inespecíficos, o que contribui para um retardo no diagnóstico. A dispneia é o sintoma mais precoce e frequente, surgindo inicialmente aos esforços e tendendo a evoluir progressivamente. Outros sintomas podem estar presentes, tais como fadiga, dor torácica, palpitações, pré-síncope e síncope (marcador de gravidade da doença). A hemoptise e a rouquidão (por compressão do nervo laríngeo recorrente por ramos dilatados da artéria pulmonar) são sintomas incomuns. ${ }^{1,2,5}$

Com o objetivo de caracterizar a intensidade da limitação funcional imposta pelos sintomas da HAP, foi criada uma classificação funcional da New York Heart Association modificada para hipertensão pulmonar (HP) pela Organização Mundial de Saúde: ${ }^{1,2,5}$

classe I: pacientes com HP, mas sem limitação das atividades físicas. Atividades físicas habituais não causam dispneia ou fadiga excessiva, dor torácica ou pré-síncope;

classe II: pacientes com HP resultando em discreta limitação das atividades físicas. Estes pacientes estão assintomáticos ao repouso, mas atividades físicas habituais causam dispneia ou fadiga excessiva, dor torácica ou pré-síncope;

classe III: pacientes com HP resultando em importante limitação das atividades físicas. Estes pacientes estão assintomáticos ao repouso, mas esforços menores que as atividades físicas habituais causam dispneia ou fadiga excessiva, dor torácica ou pré-síncope;
Quadro 1. Classificação de Dana Point (2008) para hipertensão arterial pulmonar

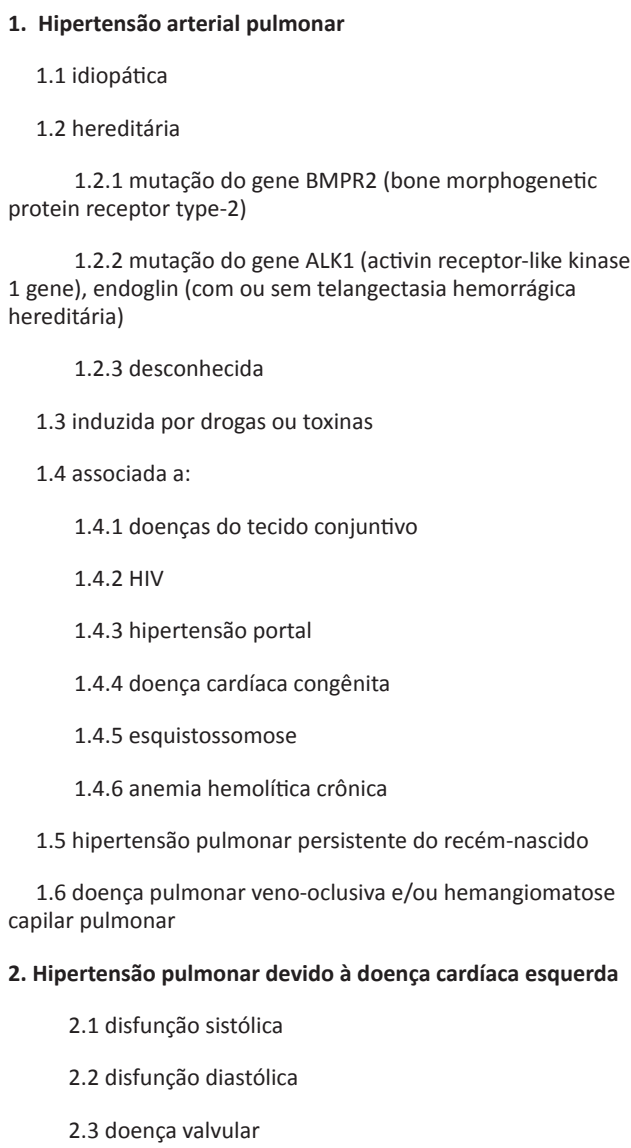

1.2.1 mutação do gene BMPR2 (bone morphogenetic protein receptor type-2)

1.2.2 mutação do gene ALK1 (activin receptor-like kinase 1 gene), endoglin (com ou sem telangectasia hemorrágica hereditária)

\subsection{3 desconhecida}

1.3 induzida por drogas ou toxinas

1.4 associada a:

1.4.1 doenças do tecido conjuntivo

1.4.2 HIV

1.4.3 hipertensão portal

1.4.4 doença cardíaca congênita

1.4.5 esquistossomose

1.4.6 anemia hemolítica crônica

1.5 hipertensão pulmonar persistente do recém-nascido

1.6 doença pulmonar veno-oclusiva e/ou hemangiomatose capilar pulmonar

2. Hipertensão pulmonar devido à doença cardíaca esquerda

2.1 disfunção sistólica

2.2 disfunção diastólica

2.3 doença valvular

3. Hipertensão pulmonar devido à doenças pulmonares e/ou hipoxemia

3.1 DPOC

3.2 doença pulmonar intersticial

3.3 outras doenças pulmonares com componente obstrutivo ou restritivo

3.4 distúrbios respiratórios do sono

3.5 síndrome de hipoventilação alveolar

3.6 exposição crônica a grandes altitudes

3.7 anormalidades do desenvolvimento pulmonar

4. Hipertensão pulmonar tromboembólica crônica

5. Hipertensão pulmonar com mecanismos multifatoriais incertos

5.1 desordens hematológicas: doença mieloproliferativa esplenectomia

5.2 desordens sistêmicas: sarcoidose, histiocitoseX, linfangioleiomiomatose, neurofibromatose, vasculites

5.3 desordens metabólicas: doença de depósito de glicogênio, doença de Gaucher, desordens tireoidianas

5.4 outros: obstrução tumoral, mediastinite fibrosante, IRC em hemodiálise 
classe IV: pacientes com HP resultando em incapacidade para realizar qualquer atividade física sem sintomas. Estes pacientes manifestam sinais de falência ventricular direita. Dispneia e/ou fadiga podem estar presentes ao repouso, e o desconforto aumenta em qualquer esforço.

\section{Exame físico}

Os achados no exame físico de um paciente com HAP idiopática variam de acordo com estágio da doença, que pode ser desde um aspecto normal até a presença de sinais clássicos de insuficiência cardíaca direita (edema de membros inferiores, hiperfonese de P2, turgência jugular, hepatomegalia e ascite) ${ }^{1,2,5,6}$

Os sinais ao exame físico podem ser didaticamente descritos como: hipertensão pulmonar, hipertrofia de ventrículo direito (VD), falência (insuficiência) de VD e baixo débito cardíaco:

a) sinais decorrentes da HAP: intensificação do componente pulmonar da segunda bulha (hiperfonese de P2), que eventualmente pode se tornar palpável. Sopro sistólico de ejeção no foco pulmonar e, nos casos mais graves, sopro diastólico de regurgitação pulmonar;

b) sinais decorrentes da hipertrofia de VD: onda A proeminente no pulso venoso jugular, presença de quarta bulha no foco pulmonar, impulsão paraesternal esquerda;

c) sinais decorrentes de falência de VD: sinais de hipertensão venosa sistêmica (turgência jugular, hepatomegalia, edema de membros inferiores e ascite), ritmo galope de B3, sopro holossistólico de regurgitação tricúspide;

d) sinais decorrentes de baixo débito cardíaco: taquicardia, vasoconstrição periférica (pulsos finos, extremidades frias e pálidas, aumento da pressão diastólica e consequente diminuição da pressão de pulso, que é a diferença entre as pressões sistólica e diastólica).

Alguns achados ao exame físico podem contribuir para identificação de eventual causa associada à HAP como, por exemplo, a presença de baqueteamento digital (incomum na HAP idiopática), que sugere associação com cardiopatia congênita. Alterações cutâneas, telangectasias e esclerodactilia impõem a investigação de doenças do tecido conjuntivo. Em pessoas obesas ou com deformidades na caixa torácica, deve ser investigada a hipoventilação como causa da HAP. ${ }^{1,2,5,6}$

Na suspeita de HAP através da anamnese e exame físico são necessárias as solicitações de exames complementares com o objetivo de confirmar diagnóstico, excluir causas associadas e avaliar a gravidade da doença. Usualmente se inicia a investigação com a realização de exames não invasivos.

\section{Exames complementares não invasivos}

- ECG: podem ser encontrados achados sugestivos, como desvio do eixo para direita (79\% dos casos), detecção de onda $\mathrm{P}$ pulmonale (aumento de sua amplitude em DII), bloqueio de ramo direito, relação R/S maior que 1 em V1, qR em V1, rSR' em V1, alteração da repolarização ventricular nas derivações precordiais direitas (figura 1).,2,5,6

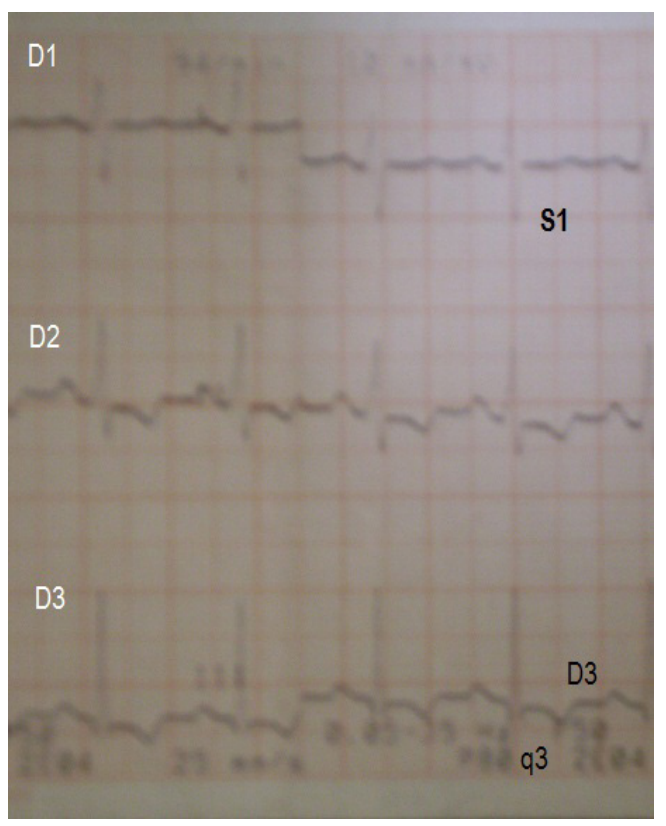

Figura 1: Eletrocardiograma com padrão de S1Q3D3 
- Radiografia de tórax: pode ser normal em pacientes assintomáticos, porém alguns achados são sugestivos de HP, como aumento no diâmetro dos ramos da artéria pulmonar, abaulamento do arco médio, pobreza vascular periférica e aumento das câmaras direitas em que, no perfil, há redução do espaço retroester- nal que passa a ser ocupado pela sombra cardíaca. É também útil para afastar diagnósticos secundários, como doenças do parênquima pulmonar ou anormalidades da caixa torácica. ${ }^{5}$ A figura 2 demonstra uma radiografia de tórax típica de hipertensão pulmonar e os aspectos tomográficos da TC na figura 3.

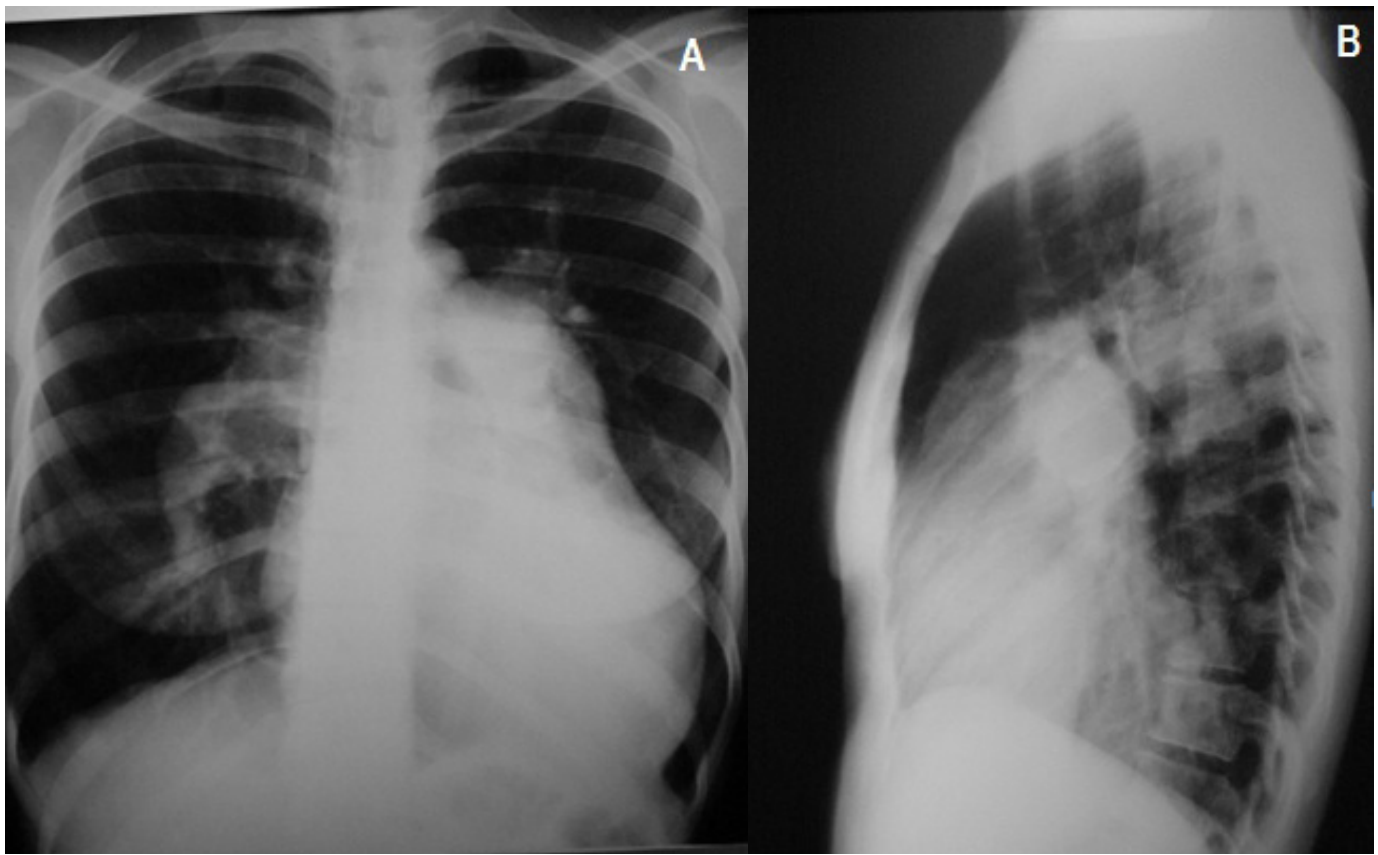

Figura 2: HAP de etiologia idiopática

Radiograma torácico posteroanterior (A) e perfil (B) com aumento importante das artérias pulmonares direita e esquerda. Há rarefação de vasos pulmonares distantes ao hilo. Aumento importante do VD com modificação da silhueta cardíaca.

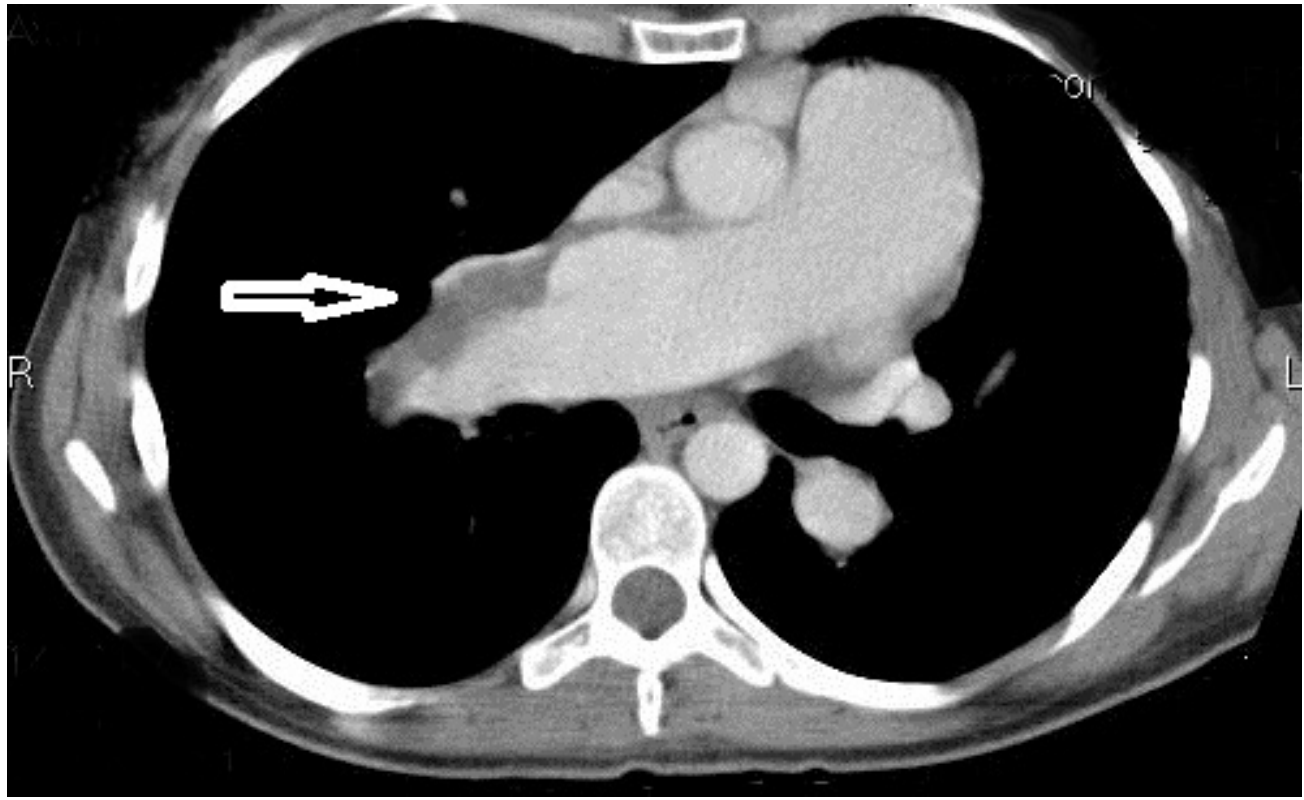

Figura 3: HAP de etiologia tromboembólica

TC de tórax com contraste. Note que o ramo direito da artéria pulmonar é mais que o dobro da aorta torácica. A seta indica a falha de preenchimento nos ramos lobares, configurando embolia pulmonar. 
O ECG e a radiografia de tórax podem trazer achados que favorecem o diagnóstico de HAP, mas têm baixa sensibilidade.

- Ecocardiograma bidimensional transtorácico com doppler: é o principal exame não invasivo para investigar a HAP, além de ser um instrumento valioso para excluir causas cardíacas associadas, como cardiopatias congênitas, disfunção de VE e doenças valvares ${ }^{5,6,7}$ A partir da presença de insuficiência tricúspide funcional, pode-se estimar a pressão sistólica da artéria pulmonar. Geralmente considera-se pressões sistólicas de artéria pulmonar acima de $40 \mathrm{mmHg}$ pelo ecocardiograma como indicativas de HAP. Os limites considerados elevados em repouso são: velocidade de regurgitação tricúspide maior que $2,8 \mathrm{~m} / \mathrm{s}$ e gradiente de pico de insuficiência tricúspide maior ou igual a $31 \mathrm{mmHg}$ (o que corresponde a PSAP 36 maior ou igual, se considerarmos $5 \mathrm{mmHg}$ de pressão atrial direita). Outros achados ecocardiográficos como dilatação do átrio direito ou do VD e achatamento, movimento paradoxal do septo interventricular, podem colaborar para reforçar a suspeita de HP. ${ }^{5,6,7,8}$
Entretanto, trata-se de um método examinador dependente e com limitações, por exemplo: a pressão de artéria pulmonar observada no ecocardiograma em repouso depende da idade, sexo, índice de massa corporal e, além disso, o aumento das pressões é observado em pessoas obesas e idosas. Por isso, para o diagnóstico definitivo, de certeza da HAP, é necessária a realização de cateterismo cardíaco direito, visto que a sensibilidade e a especificidade do ecocardiograma variam, respectivamente, entre 0,79 a 1,00 e 0,60 a $0,98 .^{1,2,5}$

Confirmada a HAP, seja com ecocardiograma com doppler (técnica não invasiva) ou com cateterismo cardíaco direito (técnica invasiva), inicia-se uma investigação complementar que tenta identificar as causas secundárias, assim como caracterizar a gravidade e programar o tratamento. ${ }^{5}$ Caso esta investigação seja negativa, está confirmada a natureza idiopática da HAP. O quadro 2 mostra a investigação mínima habitualmente recomendada e seu respectivo significado.

- Testes de função pulmonar: avaliam distúrbios ventilatórios associados a várias doenças pulmonares (DPOC, doenças intersticiais) que, aliados a hipoxemia,

\section{Quadro 2. Organograma de investigação da hipertensão arterial pulmonar}

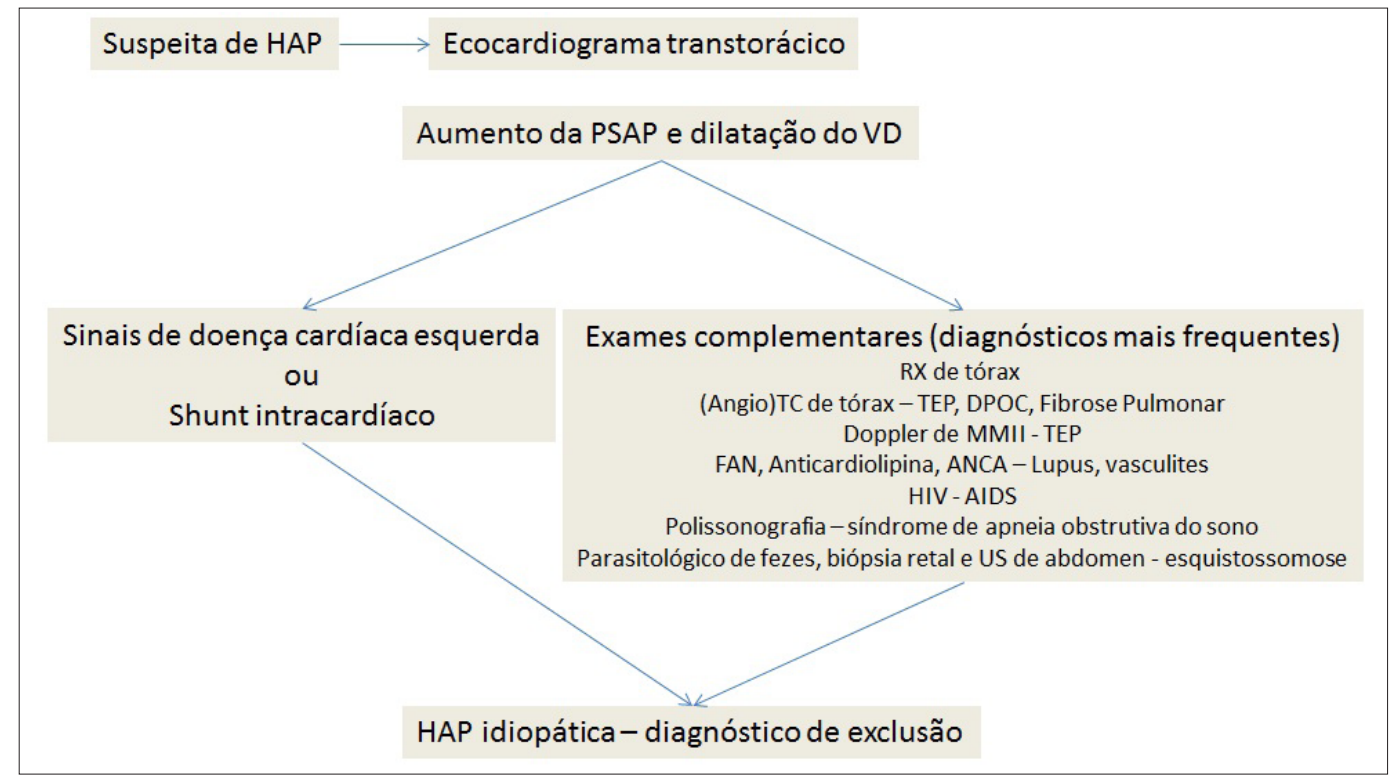

TC = tomografia computadorizada; TEP = tromboembolismo pulmonar; DPOC = doença pulmonar obstrutiva crônica. 
podem ser a causa da HAP. Pode avaliar também redução da capacidade de difusão do monóxido de carbono, que é um achado comum na HAP. ${ }^{5}$

- Tomografia computadorizada de tórax: na investigação de doenças vasculares com envolvimento parenquimatoso (vidro fosco, nódulos, espessamento de septos e consolidações). ${ }^{1,2,5}$

- Angiotomografia de tórax: para afastar tromboembolismo pulmonar agudo. ${ }^{5}$

- Cintilografia pulmonar de ventilação/perfusão: útil para afastar tromboembolismo pulmonar crônico. ${ }^{1,2,5}$

- Oximetria de pulso noturna/polissonografia: avaliação de distúrbios do sono. ${ }^{1,2,5}$

- Exames laboratoriais como o fator antinuclear (FAN), fator reumatoide (FR), ANCA (investigação de doenças do tecido conjuntivo), avaliação da função tireoidiana e pesquisa do vírus da imunodeficiência humana. ${ }^{1,2,5}$

- Pesquisa de "ovos pesados" no exame protoparasitológico de fezes, biópsia de valva retal, sorologia e ultrassonografia de abdome: em pacientes com suspeita e com epidemiologia para esquistossomose. . $^{1,25}$

- A ultrassonografia é importante também para afastar a cirrose hepática com hipertensão portal, que caracteriza a hipertensão porto-pulmonar. ${ }^{1,2,5}$

Embora formas não invasivas de avaliação da circulação pulmonar estejam cada vez mais desenvolvidas, a avaliação hemodinâmica ainda é mandatória nos quadros de HAP.

\section{Exames complementares invasivos}

A avaliação hemodinâmica da hipertensão pulmonar, realizada através do cateterismo cardíaco direito é fundamental para confirmar o diagnóstico (pressão média de artéria pulmonar acima de $25 \mathrm{mmHg}$ em repouso), investigar a etiologia, testar a vasorreatividade (que tem implicações no tratamento), estabelecer a gravidade e prognóstico, avaliar a resposta do tratamento e a progressão da doença.

Os seguintes parâmetros devem ser avaliados em condições basais no estudo hemodinâmico: frequência cardíaca, pressão média no átrio direito, pressões no ventrículo direito (sistólica e diastólica final), pressões na artéria pulmonar (sistólica, diastólica e média), débito cardíaco, pressão de oclusão da artéria pulmonar, pressão arterial sistêmica, resistência vascular pulmonar, saturação de $\mathrm{O}_{2}$ em veia cava superior, veia cava inferior, ventrículo direito, artéria pulmonar e artéria sistêmica, e resposta ao vasodilatador. . $^{5,910}$

\section{Teste da vasodilatação pulmonar (vasorreatividade)}

Estudos publicados no início da década de 1990 sugeriram que o uso crônico dos bloqueadores dos canais de cálcio (BCCa) aumentava a sobrevida daqueles pacientes com HAP que respondiam agudamente ao teste da vasodilatação pulmonar. Desde então, esse teste vem sendo utilizado com objetivo de identificar tal subgrupo de pacientes. Os que respondem satisfatoriamente ao tratamento com BCCa (em torno de 10-15\%) possuem melhor prognóstico. Em casos de instabilidade clínica ou insuficiência cardíaca direita grave, o teste não deve ser realizado devido à contraindicação dos BCCa's nessas situações. ${ }^{5,9,10}$

Os BCCa's foram os vasodilatadores mais utilizados. Entretanto, devido a sua meia-vida prolongada, a sua ação não seletiva e à ocorrência de graves efeitos adversos, atualmente os agentes de curta duração têm sido os escolhidos, minimizando assim os riscos em potencial do teste. ${ }^{5,9}$

Entre as opções de medicamentos para o teste de vasodilatação, o óxido nítrico é considerado a melhor opção e é administrado pela via inalatória na dose de 20 a 40 ppm (até 80 ppm) por 5 minutos (figura 4). Pode também ser realizado com adenosina, que é administrado em infusão venosa na dose de 50 a $250 \mathrm{mcg} /$ $\mathrm{kg} / \mathrm{min}$ (disponível no Brasil) ou com prostaciclina (epoprostenol) endovenosa. Essas duas 


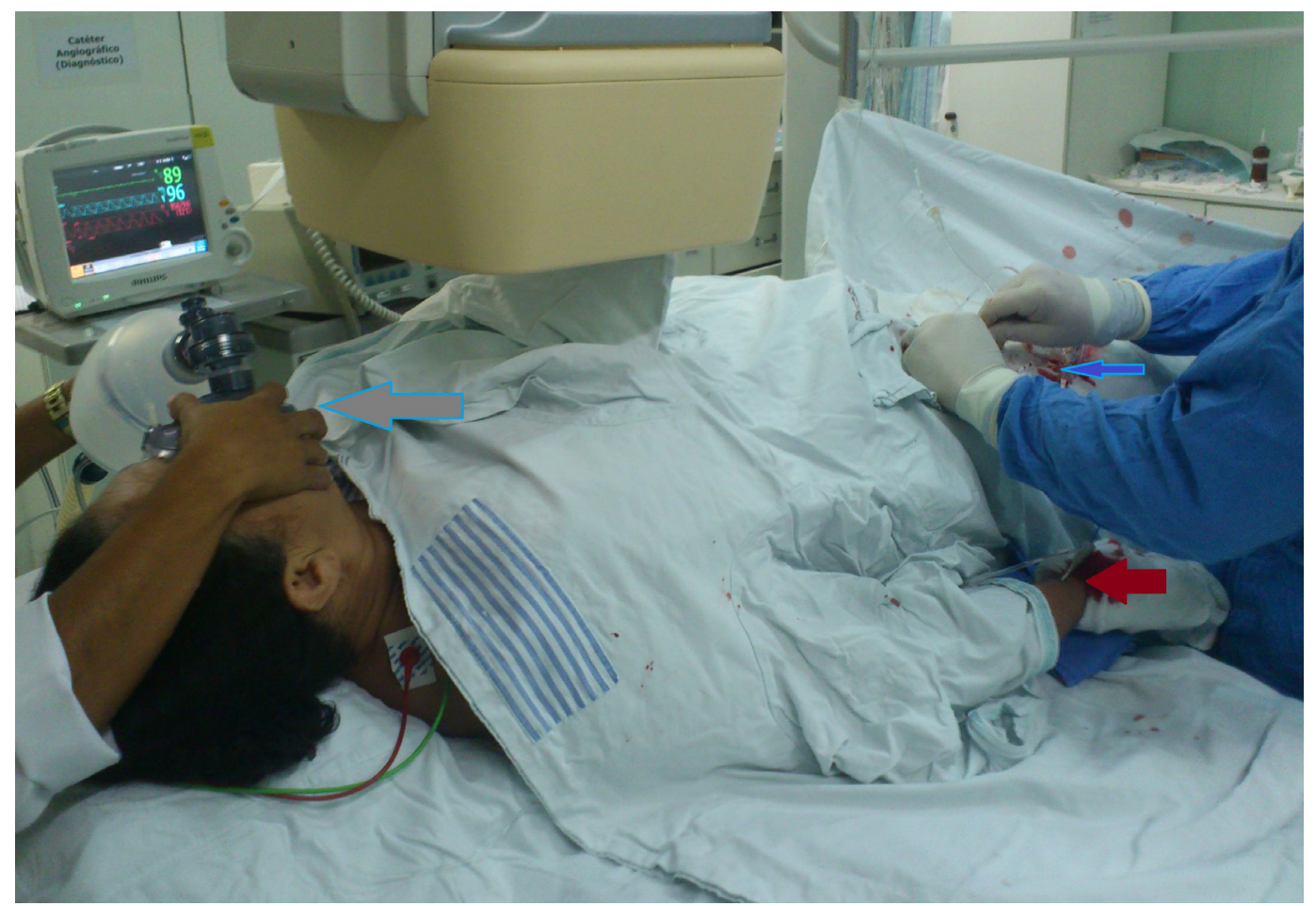

Figura 4. Teste de vasorreatividade realizado pelo setor de hemodinâmica no HUPE com óxido nítrico

A seta vermelha na artéria radial direita indica a linha arterial com um cateter que se localiza na aorta ascendente. A seta azul localiza uma linha venosa na veia femoral direita, com entrada de um cateter de Swang-Ganz para coleta e medida dos débitos e resistências cardíaca e pulmonar. A seta cinza apresenta o sistema de respiração com óxido nítrico na concentração de 10.000 ppm.

últimas causam como principal efeito colateral a hipotensão arterial sistêmica que, às vezes, pode ser refratária. Sendo assim, o óxido nítrico é a melhor opção, pois tem ação seletiva nos vasos pulmonares e curtíssima duração, o que minimiza esses efeitos e acarreta um melhor perfil de segurança. ${ }^{5,9,10}$

O teste de vasorreatividade é considerado positivo quando ocorre uma queda de mais de $10 \mathrm{mmHg}$ na pressão arterial pulmonar média e esta diminui de $40 \mathrm{mmHg}$, sem que ocorra queda no débito cardíaco. Esse teste é bem descrito e conhecido em pacientes com HAP idiopática e seu benefício é significativo, porém é importante ressaltar que apenas os pacientes que apresentarem resposta favorável ao teste de vasodilatação se beneficiarão com o uso de BCCa e são os únicos que podem ser tratados de forma segura. A administração empírica dos BCCa’s, não embasada no teste de vasorreatividade, não deve ser realizada devido à ocorrência de possíveis efeitos adversos de alta gravidade..$^{5,9,10}$

\section{Prognóstico}

Os principais marcadores prognósticos são: hemodinâmicos (índice cardíaco, pressão no átrio direito), achados ecocardiográficos (derrame pericárdico, insuficiência ventricular direita), capacidade de exercício (teste da caminhada de 6 minutos, teste de exercício cardiopulmonar com medida do consumo de $\mathrm{O}_{2}$ de pico), classe funcional de acordo com $\mathrm{New}$ York Heart Association/OMS, níveis de peptídeo natriurético cerebral (BNP)., ${ }^{1,25}$

Com base nos achados clínicos e exames não invasivos e invasivos, a condição clínica de um paciente pode ser definida como se segue (quadro 3). Pacientes nos quais todos ou a maioria dos parâmetros cumprem os critérios listados na coluna de melhor prognóstico são considerados estáveis e tratados de forma adequada. Pacientes com vários parâmetros para a coluna de pior prognóstico podem ser considerados instáveis e em fase de deteriorização, e estão em risco para agravamento da situação 
clínica. Pacientes que se enquadram no meio dessas zonas (indeterminados) são considerados como estáveis, porém não com adequado tratamento. Esses pacientes necessitam de uma reavaliação quanto à medicação adicional ou troca de medicação. A estratégia do tratamento deve ser feita de tal forma com objetivo de alcançar os parâmetros listados com grifo. ${ }^{1,2,5}$

Outros marcadores bioquímicos podem ser avaliados como a endotelina, pesquisa de dímero-D (marcador de doença trombótica), níveis séricos de ácido úrico, que se elevados demonstram alteração no metabolismo oxidativo, e a troponina T cardíaca, que em níveis elevados indica dano ao miocárdio e pode sugerir progressiva falência do ventrículo direito. ${ }^{1,2,5}$

Dentre todos esses marcadores prognósticos, a avaliação da capacidade de exercício foi utilizada como marcador principal de resposta terapêutica na maior parte dos estudos com pacientes portadores de HAP idiopática. O teste da caminhada de 6 minutos é o mais utilizado por sua capacidade prognóstica, facilidade de realização e boa reprodutilidade. ${ }^{1,2}$

\section{Tratamento}

Até a década de 1990 poucos recursos existiam para um tratamento específico desta doença de prognóstico reservado. Porém o desenvolvimento de novas alternativas terapêuticas, tendo como base a fisiopatologia da HAP, possibilitou uma mudança significativa na evolução desses pacientes. Essa mudança gerou não só uma maior qualidade de vida, mas também um aumento da sobrevida. . $^{2,511}$

O tratamento ideal para um paciente é a tomada de decisão altamente individualizada que leva em consideração muitos fatores como: gravidade da enfermidade, via de administração, efeitos colaterais, comorbidades, preferência do especialista e metas terapêuticas. Os principais objetivos do tratamento são a manutenção do paciente em classe funcional I ou II, um exame físico sem sinais de falência de VD, teste da caminhada de 6 minutos acima de 400 metros e um BNP próximo do normal, estável ou em declínio. ${ }^{1,2,5,11}$

A vasoconstrição, o remodelamento da parede dos vasos pulmonares e a trombose in situ são fatores envolvidos no aumento da resistência vascular pulmonar na HAP. Porém, acredita-se que o remodelamento vascular pulmonar seja o principal determinante da patogenia e da evolução da HAP. ${ }^{1,2,5,11}$

A abordagem terapêutica se baseia no tratamento dos fenômenos associados e na dilatação e antirremodelação vascular pulmonar.

\section{Quadro 3. Avaliação prognóstica na hipertensão arterial pulmonar}

\begin{tabular}{|c|c|c|}
\hline Melhor prognóstico (estável) & Critérios de prognóstico & Pior prognóstico (instável) \\
\hline Não & $\begin{array}{l}\text { Evidências clínicas de insuficiência } \\
\text { ventricular direita }\end{array}$ & Sim \\
\hline Não ou devagar & Taxa de progressão da doença & Rápido \\
\hline Não & Síncope & Sim \\
\hline I, II & Classe funcional & IV \\
\hline Mais de $400-500 \mathrm{~m}^{*}$ & Teste da caminhada dos 6 minutos & Menor que $300 \mathrm{~m}$ \\
\hline Pico $\mathrm{VO}_{2}>1 \mathrm{ml} / \mathrm{min} / \mathrm{kg}$ & Teste de exercício cardiopulmonar & $\mathrm{Pico} \mathrm{VO}_{2}<12 \mathrm{ml} / \mathrm{min} / \mathrm{kg}$ \\
\hline Normal ou perto do normal & Níveis séricos de BNP/NT pro BNP & Muito elevado e/ou em ascensão \\
\hline Sem derrame pericárdico TAPSE $>2 \mathrm{~cm}$ & Achados ecocardiográficos & Derrame pericárdico TAPSE $<1,5 \mathrm{~cm}$ \\
\hline $\begin{array}{c}P A D<8 \mathrm{mmHg} e \\
\text { IC }>\text { ou igual } 2,5 \mathrm{I} / \mathrm{min} / \mathrm{m}^{2}\end{array}$ & Hemodinâmica & $\begin{array}{c}\mathrm{PAD}>15 \mathrm{mmHg} \text { ou } \\
\mathrm{IC}<\text { ou igual } 2 \mathrm{I} / \mathrm{min} / \mathrm{m}^{2}\end{array}$ \\
\hline
\end{tabular}

BNP: peptídeo natriurético cerebral; IC: índice cardíaco; pico $\mathrm{VO}_{2}$ : captação máxima de oxigênio; PAD: pressão átrio direito; TAPSE: tricuspid anular plane systolic excursion (movimentação plana da valva tricúspide durante a sístole).

* Depende de variáveis como idade, peso, condições físicas, comorbidades. TAPSE e derrame pericárdico foram selecionados porque podem ser medidos na maioria dos pacientes. 


\section{Tratamento dos fenômenos} associados

- Anticoagulação: achados de lesões trombóticas em necropsias de pacientes com HAP, descrições de anormalidades no sistema fibrinolítico, presença de fatores de risco para trombose in situ (diminuição do fluxo sanguíneo pulmonar ou estado de hipercoagulabilidade observado nesses pacientes e outros fatores de risco não específicos como insuficiência cardíaca e imobilidade) acabam constituindo, em conjunto, o racional para a anticoagulação desses pacientes, desde que não haja contraindicação. A anticoagulação é feita com objetivo de manter o INR entre 1,5 e 2,5. $2,5,11$

- Diuréticos: controle do edema devido ao aumento do volume intravascular dos pacientes com disfunção ventricular direita grave e/ou uso de altas doses de BCCa. Deve ser usado com cautela para evitar a diminuição excessiva da pré-carga e também o aumento da viscosidade sanguínea, com consequente aumento do risco de trombose. Monitoração de eletrólitos, função renal e equilíbrio ácido-base deve ser feita nesses pacientes. ${ }^{2,5,11}$

- Digital: o uso da digoxina na HAP idiopática ainda é controverso. Embora alguns estudos tenham evidenciado efeitos benéficos como a diminuição do nível sérico das aminas simpaticomiméticas, seus benefícios clínicos não são totalmente comprovados. Tem sido mais usada quando há falência de VE concomitante ou para controle de taquicardias supraventriculares. Atentar para sinais de intoxicação digitálica. ${ }^{2,5,11}$

- Oxigenioterapia: indicado em pacientes com $\mathrm{SatO}_{2}$ menor que $88 \%$ por pelo menos 15 horas por dia, com objetivo de mantê-la acima de $90 \%{ }^{2,5,11}$

\section{Tratamento da dilatação e} antirremodelação vascular pulmonar

Como já dito anteriormente, a vasoconstrição pulmonar é um dos mecanismos envolvidos na patogenia da HAP, com hipertofia da camada média, diminuição da produção de vasodilatadores pelo endotélio pulmonar (prostaciclinas e óxido nítrico) e aumento da produção de vasoconstritores (endotelina). As principais medicações são:

bloqueadores de canal de cálcio - sua indicação é reservada para os pacientes com classe funcional III ou IV e que apresentam resposta positiva ao teste de vasodilatação pulmonar. Pacientes não submetidos ao teste ou cujo o mesmo foi negativo não devem receber tal medicação pelo risco de efeitos colaterais graves como hipotensão, síncope e morte súbita. Os BCCa’s utilizados são a nifedipina (30 a $240 \mathrm{mg} / \mathrm{dia}$ ), diltiazem (120 a $720 \mathrm{mg} /$ dia) e anlodipina (2,5 a $20 \mathrm{mg} / \mathrm{dia})$. Mesmo nos pacientes com resposta positiva ao teste, o benefício da manutenção do BCCa deve ser sempre reavaliado, devendo considerar outras opções terapêuticas caso a resposta não seja mantida; $2,5,11$

inibidores da fosfodiesterase 5 - os 3 inibidores da fosfodiesterase tipo 5 usados para tratamento da disfunção erétil (sildenafil, tadalafil e vardenafil) aumentam o GMP cíclico, prolongando o efeito vasodilatador do óxido nítrico. Além de promoverem a vasodilatação pulmonar, eles têm ainda efeito antiproliferativo, mas a maior experiência clínica no tratamento da HP é com sildenafil na dose habitual de $20 \mathrm{mg}$ a cada 8 horas. Estudos mostram que ele é que determina melhora clínica, funcional e hemodinâmica. ${ }^{2,5,11}$ Nas diretrizes atuais, o sildenafil constitui-se uma das primeiras opções para tratamento de pacientes em classe funcional II ou III. Nos pacientes em classe funcional IV, a recomendação de tratamento inicial é com epoprostenol, mas como este não está disponível no Brasil, o sildenafil passa a ser também uma opção; ;,5,11

- derivados da prostaciclina: são potentes vasodilatadores, inibem a agregação pla- 
quetária e diminuem a proliferação das células musculares lisas. Devido a essas propriedades, têm sido recomendados no tratamento da HAP, mas seu uso é limitado em função de alto custo e dificuldades de administração. Atualmente existem 3 drogas derivadas da prostaciclina para o tratamento da HAP, porém nenhuma disponível no Brasil. São elas: epoprostenol, treprostinil e iloprost. ${ }^{2,5,11} \mathrm{O}$ epoprostenol, por possuir meia-vida curta (3-5 minutos) deve ser administrado por via endovenosa (cateter venoso central de longa permanência), de forma contínua através de bomba de infusão. Embora estudos tenham mostrado melhora na sobrevida com esta medicação, o custo e as possíveis complicações limitam seu uso. ${ }^{2,5,11} \mathrm{O}$ treprostinil é um análogo da prostaciclina e é usado via subcutânea. Estudos mostraram eficácia inferior ao epoprostenol. A dor no local da infusão pode ser fator limitante para seu uso. ${ }^{2,5,11} \mathrm{O}$ iloprost é um análogo estável da prostaciclina e o fato de ser usado via inalatória minimiza seus efeitos de vasodilatação sistêmica. Estudos mostraram que em pacientes com HAP nas classes funcionais III e IV ele foi capaz de melhorar a capacidade de exercício e dispneia. No entanto, a necessidade de inalações repetidas (6 a 9 vezes ao dia) e uso de nebulizadores específicos são fatores limitantes ao seu uso; $;, 2,11$

bloqueadores dos receptores de endotelina: a endotelina é um potente vasoconstritor, ativando-se ao ligar aos seus receptores (ET-A e ET-B) encontrados nas células endoteliais e células musculares lisas vasculares. Além de vasoconstritor, a endotelina tem efeito mitógeno e fibrogênico. Essas medicações têm sido usadas com bons resultados na HAP. São elas: bosentana, sitaxsentana e ambrisentana. ${ }^{2,5,11} \mathrm{~A}$ bosentana é um antagonista não seletivo dos receptores da endotelina (A e B), é usada na dose de $62,5 \mathrm{mg}$, duas vezes ao dia por 4 semanas e, a seguir, $125 \mathrm{mg}$, duas vezes ao dia. Com seu uso, há uma melhora da capacidade ao exercício, da hemodinâmica, do grau de dispneia e aumento da sobrevida em pacientes com HAP idiopática. A grande vantagem é a sua administração via oral e baixa toxicidade, porém deve-se monitorizar a função hepática e pode interferir com uso de varfarina. ${ }^{2,5,11}$

A sitaxsentana é um antagonista seletivo do receptor ET-A da endotelina, usada na dose de $100 \mathrm{mg} / \mathrm{dia}$. Com seu uso pode haver interação com varfarina e também se deve monitorizar a função hepática., ${ }^{2,5,11}$ A ambrisentana também é um antagonista seletivo do receptor ET-A e é usada na dose de 5 a $10 \mathrm{mg} /$ dia. $^{2,5,11}$

\section{Terapia combinada}

O termo 'terapia combinada' refere-se ao emprego de medicações de mais de uma classe, dentre as disponíveis para tratamento da HAP. Como elas atuam em sítios diferentes a associação faz sentido, sobretudo em pacientes sem resposta clínica adequada com monoterapia. No nosso meio, a combinação disponível é entre um inibidor de fosfodiesterase tipo 5 e um antagonista de endotelina, mas vários pontos ainda merecem estudos em relação à terapia combinada. $^{12}$

\section{Tratamento cirúrgico}

Para os pacientes refratários a todas as medidas farmacológicas, existem atualmente duas alternativas cirúrgicas no tratamento da HAP idiopática: a septostomia atrial e o transplante pulmonar. $^{2}$

A septostomia atrial é considerada uma alternativa terapêutica para locais sem acesso às drogas vasodilatadoras e com programas de transplantes pulmonares ainda incipientes. A septostomia atrial pode ser utilizada como uma ponte para a realização do transplante, com melhora funcional dos pacientes. Portanto, deve-se ressaltar que a indicação da septostomia atrial segue critérios específicos e deve ser realizada 
apenas em centros com experiência, uma vez que a taxa de mortalidade do procedimento é alta. ${ }^{2}$

Os critérios de indicação do transplante pulmonar na HAP idiopática estão sendo revistos em função do advento de novas drogas para o tratamento dessa entidade. O paciente deve ser encaminhado para centros de referência em hipertensão pulmonar para que a avaliação conjunta possa determinar os critérios de falência de tratamento. Determinada a falência do tratamento clínico, o paciente deve ser encaminhado para seguimento em um centro de transplante pulmonar. ${ }^{2}$

A taxa de sobrevida após transplante por HAP, em 3 anos, é de aproximadamente 65 a 70\%.

$\mathrm{O}$ tratamento da HAP baseado em evidências, de acordo com o IV Simpósio de HAP, realizado em Dana Point (EUA), está descrito no quadro $4 .^{2}$

\section{Monitorização da hipertensão arterial pulmonar}

Por se tratar de uma doença grave e progressiva, estes pacientes devem ser acompanhados rigorosamente no sentido de se perceber rapidamente aqueles que estão deteriorando ou não respondendo ao tratamento para que este possa ser ajustado. Os parâmetros que devem ser avaliados são: dados clínicos, classe funcional, ECG, teste da caminhada de 6 minutos, teste de exercício cardiopulmonar, ecocardiograma, BNP, cateterismo direito e ressonância nuclear magnética. Esta última fornece uma melhor avaliação do tamanho e função do VD, quando comparada ao ecocardiograma. ${ }^{1,2,5,11}$

\section{Medidas recomendadas para pacientes com HAP}

- Mulheres com HAP devem evitar gravidez.

Quadro 4. Algoritmo de Tratamento de HAP baseado em evidências - IV Simpósio de HAP, 2008

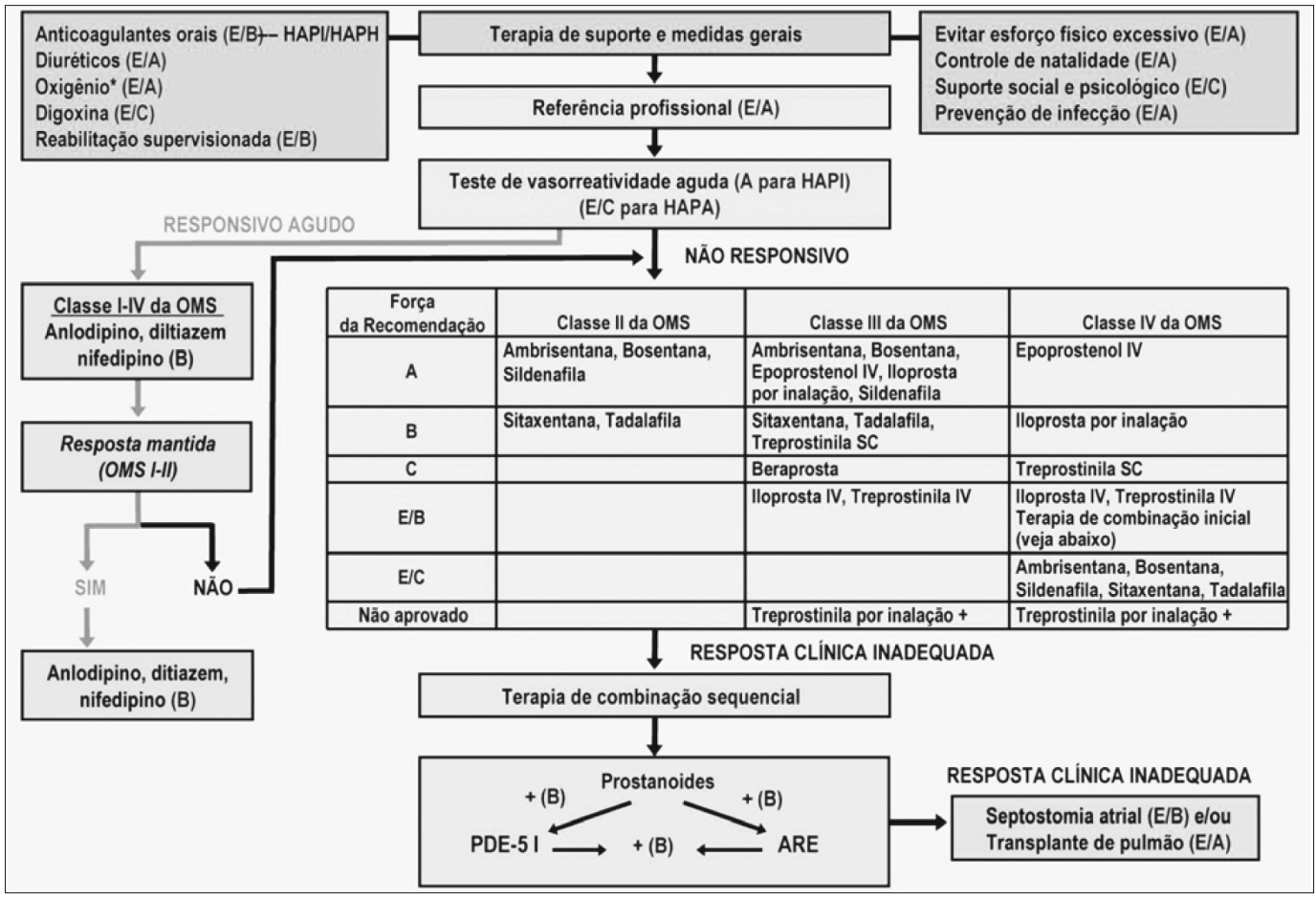

Os medicamentos dentro do mesmo grau de evidência estão listados em ordem alfabética e não por ordem de preferência. Nem todos os agentes listados estão aprovados ou disponíveis para uso em todos os países.

HAPA = hipertensão arterial pulmonar associada; ARE = antagonista do receptor da endotelina; HAPH = hipertensão arterial pulmonar hereditária; HAPI = hipertensão arterial pulmonar idiopática; IV = intravenosa; HAP = hipertensão arterial pulmonar; PDE 5 = fosfodiesterase tipo 5; SC = subcutânea; OMS = Organização Mundial da Saúde. 
A mortalidade chega a $30-50 \%$. Deve-se pesar a eficácia, riscos potenciais (ex.: efeito trombogênico dos estrógenos) e interações medicamentosas (ex.: bosentana diminui a eficácia dos anticoncepcionais orais). ${ }^{1,2,5,11}$

- Vacinação contra influenza e pneumococo. ${ }^{1,2,5,11}$

- Manutenção de atividade física que não determine dispneia. Pacientes sedentários devem ser encaminhados a programas de reabilitação. ${ }^{1,2,5,11}$

- Pacientes que fazem uso de oxigenioterapia, em caso de viagens aéreas, devem receber suplementação de $\mathrm{O}_{2}$ durante o voo. . $2,5,11^{-1}$

- Em cirurgias eletivas, o risco cirúrgico será sempre elevado. Deve-se optar por anestesias com bloqueios, como raqui ou peridural. ${ }^{1,2,5,11}$

- Suporte psicológico. ${ }^{1,2,5,11}$

\section{Referências}

1. Grunig E, Barner A, Bell M, Claussen M, Dandel M, Dumitrescu D, et al. Non-invasive diagnosis of pulmonary hypertension:ESC/ERS Guidelines with Uptaded Commentary of the Cologne Consensus Conference. Int J Cardiol. 2011;154 (Suppl 1):3-12.

2. Ghofrani HA, Distler O, Gerhardt F, Gorenflo M, Grunig E, Haefeli WE, et al. Treatment of pulmonary arterial hypertension(PAH):Updated Recommendations of the Cologne Consensus Conference. Int J of Cardiol. 2011;154(Suppl1):20-33.

3. Ventetuolo CE, Klinger JR. WHO Group 1 pulmonary arterial hypertension: current and investigative therapies. Prog Cardiovasc Dis. 2012;55(2):89-103. http://dx.doi.org/10.1016/j. pcad.2012.07.002.

4. Chan SY, Loscalzo J. Pathogenic mechanisms of pulmonary arterial hypertension. J Mol Cell Cardiol. 2008;44(1):14-30.

5. SBPT. Diretrizes Brasileiras para Manejo da Hipertensão Pulmonar. J Bras Pneumol. 2005; 31:1-23.

6. Vachiéry JL, Gaine S.Challenges in the diagnosis and treatment of pulmonary arterial hypertension. Eur Respir Rev. 2012 1;21(126):313-20.

7. LancellottiP, BudtsW, DeWolfD, VoigtJU, Pasquet A, De Sutter J, et al. Practical recommendations on the use of echocardiography to assess pulmonary arterial hypertension--a Belgian expert consensus endorsed by the Working Group on Non-Invasive Cardiac Imaging. Working Group on Non-Invasive Cardiac Imaging. Acta Cardiol. 2013;68(1):59-69.

8. Bossone E, D'Andrea A, D'Alto M, Citro R, Argiento P, Ferrara F, et al. Echocardiography in pulmonary arterial hypertension: from diagnosis to prognosis. J Am Soc Echocardiogr. 2013;26(1):1-14.

9. Lau EM, Manes A, Celermajer DS, Galiè N. Early detection of pulmonary vascular disease in pulmonary arterial hypertension: time to move forward. Eur Heart J. 2011; 32(20):2489-98.

10. Vachiéry JL, Yerly P, Huez S. How to detect disease progression in pulmonary arterial hypertension. Eur Respir Rev. 2012;123:40-47.

11. Rosenblum WD. Pulmonary arterial hypertension: pathobiology, diagnosis, treatment, and emerging therapies. Cardiol Rev. 2010;18(2):58-63.

12. Meis T, Behr J. Pulmonary hypertension: role of combination therapy. Curr Vasc Pharmacol. 2011;9(4):457-64. 